\title{
Magnetic Decreases (MDs) and mirror modes: two different plasma $\beta$ changing mechanisms
}

\author{
B. T. Tsurutani ${ }^{1,2}$, G. S. Lakhina ${ }^{3}$, O. P. Verkhoglyadova ${ }^{1,4}$, E. Echer $^{5}$, and F. L. Guarnieri ${ }^{6}$ \\ ${ }^{1}$ Jet Propulsion Laboratory, California Institute of Technology, Pasadena, CA, USA \\ ${ }^{2}$ Institut für Geophysik und Extraterrestrische Physik, Technische Universität Braunschweig, Braunschweig, Germany \\ ${ }^{3}$ Indian Institute of Geomagnetism, Navi Mumbai, India \\ ${ }^{4}$ CSPAR, University of Alabama, Huntsville AL, USA \\ ${ }^{5}$ Instituto Nacional Pesquisas Espaciais, São Jose dos Campos, SP, Brazil \\ ${ }^{6}$ Universidade do Vale do Paraíba, São Jose dos Campos, SP, Brazil
}

Received: 6 June 2010 - Revised: 15 August 2010 - Accepted: 18 August 2010 - Published: 27 September 2010

\begin{abstract}
We discuss two different physical processes that create localized high $\beta$ plasma regions. One is nonlinear wave-steepening, generating magnetic decreases (MDs) by a ponderomotive force. The other is the mirror instability generating alternating high and low $\beta$ plasma regions. It is demonstrated that MDs and mirror modes are observationally quite different structures. MDs spatially occur in interplanetary space and mirror modes primarily in planetary magnetosheaths. MDs are characterized by: 1) variable (exponentially decreasing number with increasing) angular changes, 2) variable (exponentially decreasing) thicknesses, and 3) no characteristic inter-event spacings. In sharp contrast, mirror modes are characterized by: 1) little or no angular changes across the structures, 2) a characteristic scale size, and 3) are quasiperiodic in nature.

Arguments are presented for the recently observed magnetic dips in the heliosheath being mirror mode structures. The sources of free energy for instability are discussed. Both structures are important for energetic particle transport in astrophysical and heliospheric plasmas.
\end{abstract}

\section{Introduction}

\section{1 "Magnetic holes"}

The term "magnetic hole" was first introduced into the literature by Turner et al. (1977). It was used to describe deep decreases (dips) in the interplanetary magnetic field magni-

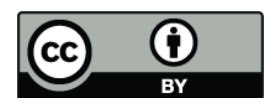

Correspondence to: B. T. Tsurutani (bruce.tsurutani@jpl.nasa.gov) tude. Tsurutani and Ho (1999) called Ulysses high latitude magnetic dips "magnetic decreases" and Fränz et al. (2000) called these structures "magnetic depressions", mostly because at the time it was not certain whether the structures from the different studies and different physical locations in space were the same or not. It has been since concluded that these structures are most probably the same phenomenon (Tsurutani et al., 2002a) and will be discussed as such in this paper.

Recently, magnetic dips have been detected by the Voyager spacecraft in the heliosheath (Burlaga et al., 2006, 2007). Burlaga et al. (2006) have called these structures "magnetic holes" as a descriptive term (L. F. Burlaga, private communication, 2009). Burlaga did not intend for readers to assume that magnetic dips in interplanetary space and in the heliosheath were necessarily generated by the same physical process. For the sake of avoiding confusion, we will call the interplanetary structures magnetic decreases or MDs, and will investigate what the heliosheath structures are. We will show that the heliosheath structures are different and are generated by a different mechanism, the mirror instability.

\subsection{Mirror mode structures}

The mirror instability was first theoretically investigated by Chandrasekar et al. (1958) and Vedenov and Sagdeev (1958) and later clarified and elucidated by Hasegawa $(1969,1975)$. The expression for instability is Eq. (1):

$\beta_{\perp} / \beta_{\|}>1+1 / \beta_{\perp}$

where $\beta$ is the thermal plasma pressure divided by the magnetic pressure and the subscripts $\perp$ and $\|$ correspond to the components perpendicular and parallel to the ambient

Published by Copernicus Publications on behalf of the European Geosciences Union and the American Geophysical Union. 
magnetic field $B_{0}$, respectively. Price et al. (1986), Southwood and Kivelson (1993), and Pokhotelov et al. (2004) are more recent theoretical investigations of the mirror instability and its features and we refer the reader to those articles for more details about the instability. The first detailed observations in space plasmas and correct identification of this instability were made by Tsurutani et al. (1982). They established that the mirror mode instability was operative in the planetary magnetosheaths of the Earth, Jupiter and Saturn. More importantly, they and the results of computer simulations of Price et al. (1986) established the relative phasing magnetic and plasma features of mirror mode structures, the quasiperiodic nature of these structures, and their convection/propagation in magnetosheath plasmas. One particular feature of importance is that the magnetic field directionality varies only slightly across the mirror mode structures (Tsurutani et al., 1982).

Although 85-90\% of MDs have substantial magnetic field angular changes across them, there are $\sim 15 \%$ that have angular changes $<10^{\circ}$ ("linear" structures). Winterhalter et al. (1994) noticed that the magnetic field pressure within the MD dips was supplanted by plasma pressure (so that total pressure was constant, to first order) and that the mirror instability criterion was met. Thus they speculated that at least the small portion of the MDs ( 10-15\%) might have been generated by the mirror instability. However, they did not and could not rule out the possibility that other mechanisms might cause the high plasma beta regions. Winterhalter et al. (1994) also did not have an explanation for the other 85-90\% of MDs that were not "linear". They also did not have an answer for a mechanism that could lead to plasma anisotropy and mirror instability.

Thus, for several historical reasons, the distinction between interplanetary MDs and magnetosheath mirror modes has been blurred. Part of the purpose of this paper is to review what is known and not known about interplanetary MDs and the magnetosheath structures so that confusion will be minimized and that future researchers will be able to make progress to better understand these very interesting plasma features.

A second emphasis of this paper is to demonstrate that nonlinear wave processes can and do change the local plasma $\beta$. We will show several examples of this in our review. This is an important feature of plasma turbulence and should be incorporated in astrophysical plasma models, where appropriate. This paper was given as a talk at the 8th International Nonlinear Wave Workshop in La Jolla, California, 1-5 March 2010.

\section{Results}

\subsection{Magnetic decreases}

\subsubsection{Magnetic Decrease (MD) formation in the freely propagating solar wind}

Figure 1 shows 30 days of solar wind magnetic field and velocity components when Ulysses was over the Sun's poles. The RTN coordinate system is called solar heliospheric (SH), where $R$ is the radial direction from the sun to the spacecraft, $T=(\hat{\Omega} \times R) /|\hat{\Omega} \times R|$, where $\hat{\Omega}$ is the north rotation pole of the sun, and $N$ completes a right-hand coordinate system. The seventh panel from the top shows the nearly constant, high speed solar wind emanating from the polar region of the sun. During this phase of the solar cycle (the declining phase), large polar coronal holes existed at the sun. The high speed solar wind came from these holes (Phillips et al., 1995).

The large fluctuations in the magnetic field and solar wind velocity components (top six panels) are large-amplitude, nonlinear Alfvén waves (see discussion in Belcher and Davis, 1971; Tsurutani et al., 1994). These waves are propagating outward from the sun (Balogh et al., 1995), but because the solar wind convection speed is much larger than the Alfvén wave phase speed, the waves are mostly convected radially outward from the sun. The peak-to-peak wave amplitude $\Delta b$ can be as large as $1-2$ times the field magnitude $B_{0}$.

The magnetic field magnitude is given in the next-to-bottom panel (the Ulysses polar passes occurred at $r \sim 2 \mathrm{AU}$ from the sun). Note that there are many sharp magnetic field magnitude decreases (MDs) that appear as spikes on this compressed time scale. As previously mentioned, the MDs are pressure balance structures, where the total pressure, i.e., plasma pressure $\left(\Sigma_{i} n_{i} k T_{i}\right)$, plus magnetic pressure $\left(B^{2} / 8 \pi\right)$ is constant across the structures (Winterhalter et al., 1994). In the above expression for the plasma pressure, $n_{i}$ and $T_{i}$ are the densities and temperatures of the $i$-th species (electron, proton and ion), and $k$ is the Boltzmann constant.

It should be noted that although there are many MDs present in the figure, there are very few comparable positive magnetic spikes. This will be discussed later in the paper.

What do MDs look like in higher resolution? Figure 2 shows 3 examples of MDs in the Ulysses data taken at $\sim 2 \mathrm{AU}$ from the sun. In each of the examples, the panels give the $r$, $t$ and $n$ components and $B_{0}$ the magnitude of the magnetic field. Vertical dashed lines border the beginnings and ends of the MDs.

Each of the 3 MDs have different magnetic field directional changes across the structures. The top example has little directionality change (except some in $B_{r}$ ). The second example has both large component changes and $B_{0}$ changes across it. The third panel shows an event with a sharp 


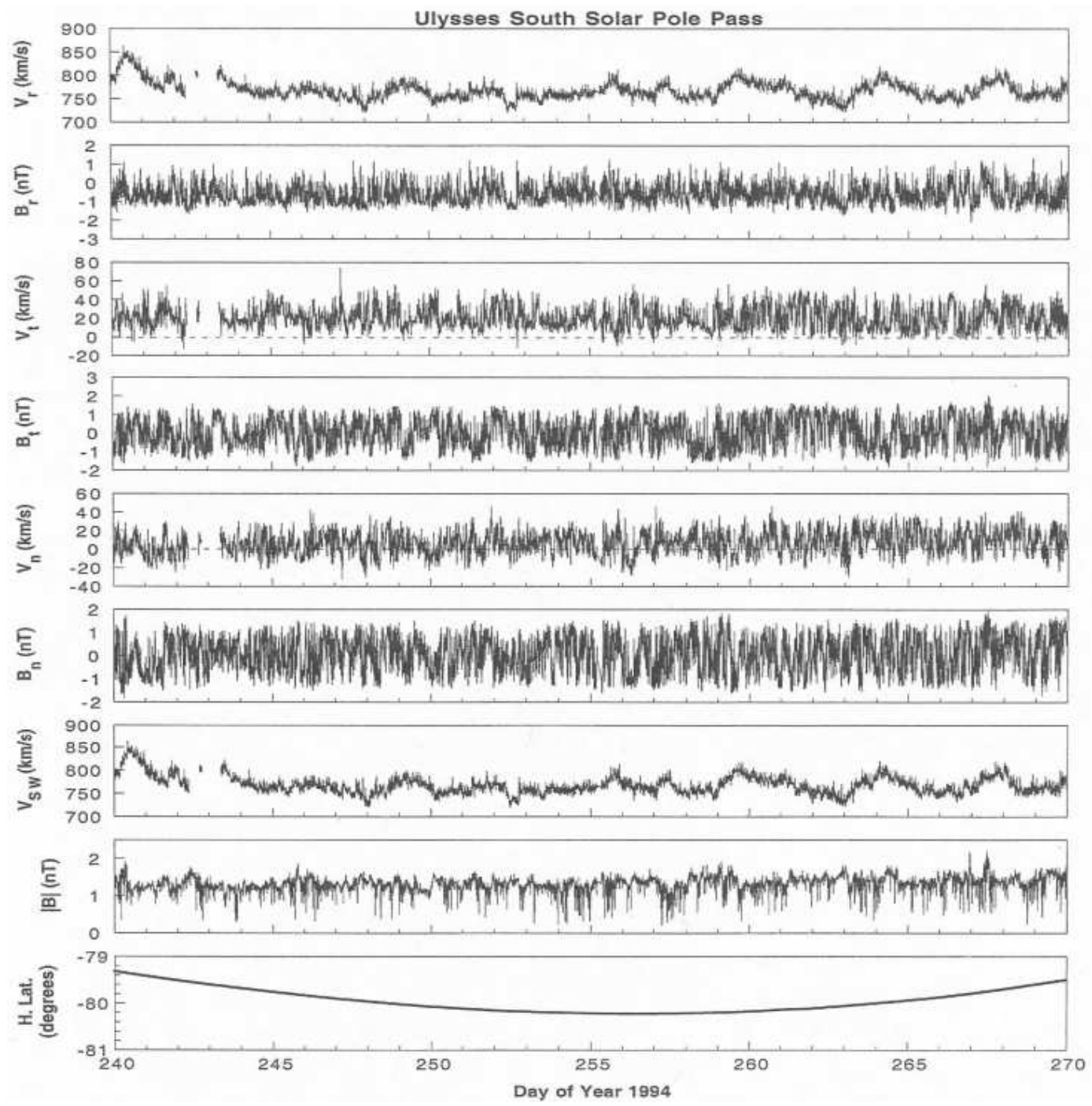

Fig. 1. The Ulysses velocity and magnetic field components for 30 days over the south pole of the sun. MDs are the sharp field magnitude decreases noted in the next to last panel. Taken from Tsurutani and Ho (1999).

component change $\left(B_{n}\right)$ coincident with the edge of the MD. All three of these examples have sharp $B_{0}$ boundaries at the edges of the MDs.

Figure 3 illustrates the detailed relationship between Alfvén waves and MDs. The top panel shows three cycles of an Alfvén wave. The magnetic field components are given in the minimum variance coordinates where the subscripts 1 , 2 and 3 correspond to the maximum, intermediate and minimum variance directions, respectively. The field magnitude is shown at the bottom. The bottom panel gives the wave hodogram for the middle wave cycle, from points 1 through 3. The Alfvén wave slowly rotates (in phase) from points 1 to 2 and then rotates rapidly from points 2 to 3 . This effect is called "phase-steepening", indicating that the wave is not sinusoidal in nature, but has a steepened edge. Presumably nonlinear effects cause the Alfvén wave phase-steepening
(Hada, 1993; Medvedev et al., 1997; Buti et al., 2001). All three wave cycles are phase-steepened.

Dips in the magnetic field magnitude (MDs) are noted at the phase-steepened edges of the 3 wave cycles shown in the figure. It has been speculated by Tsurutani et al. (2002a) and Dasgupta et al. (2003) that ambipolar acceleration of protons occurs through the ponderomotive force associated with the phase-steepened edges of the Alfvén waves. The heating of the ions (and electrons) create the magnetic dips through the diamagnetic effect of the perpendicularly heated plasma. Evidence for local heating of the ions and plasma instabilities have been noted, but will not be discussed here for brevity. The interested reader is referred to Tsurutani et al. (2005a) and references therein.

The hypothesis that the MDs are formed by a diamagnetic effect associated with the creation of a hot plasma region can 
a)

ठ্م

b)

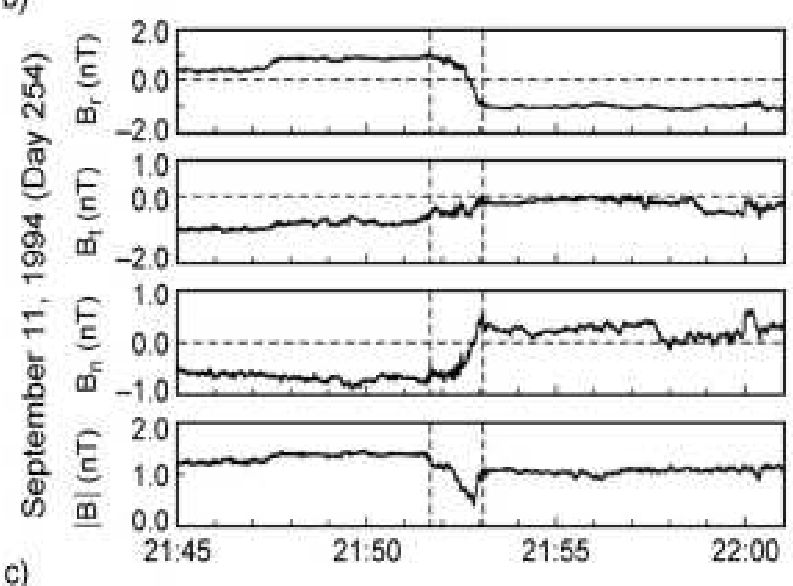

c)

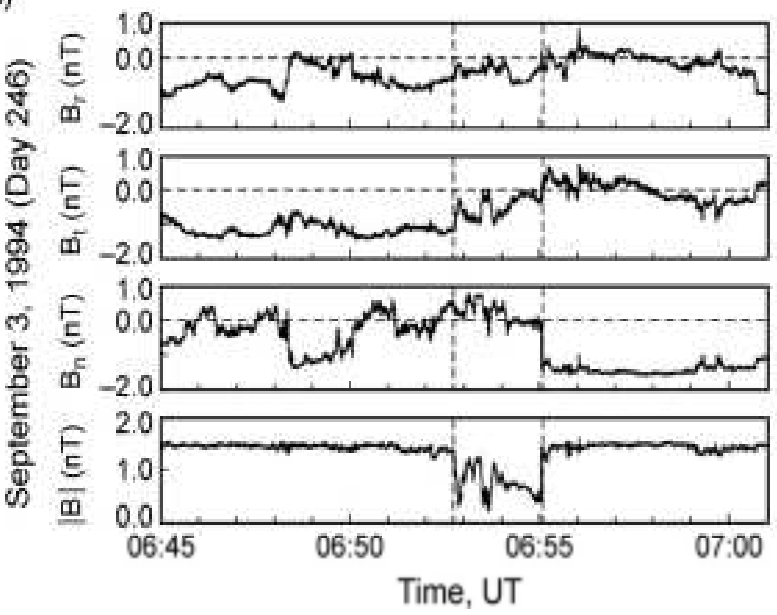

Fig. 2. Three examples of MDs in the Ulysses data. The field components are in heliospheric RTN coordinates.

explain the absence of enhanced fields as well. The local expulsion of the magnetic field from within the plasma current loops will displace these fields to larger distances outside the loops. Thus the field increases are expected to be present, but occur gradually in regions outside the MDs.

The previous discussion showed that MDs form in association with the phase-steepening of Alfvén waves. In the high
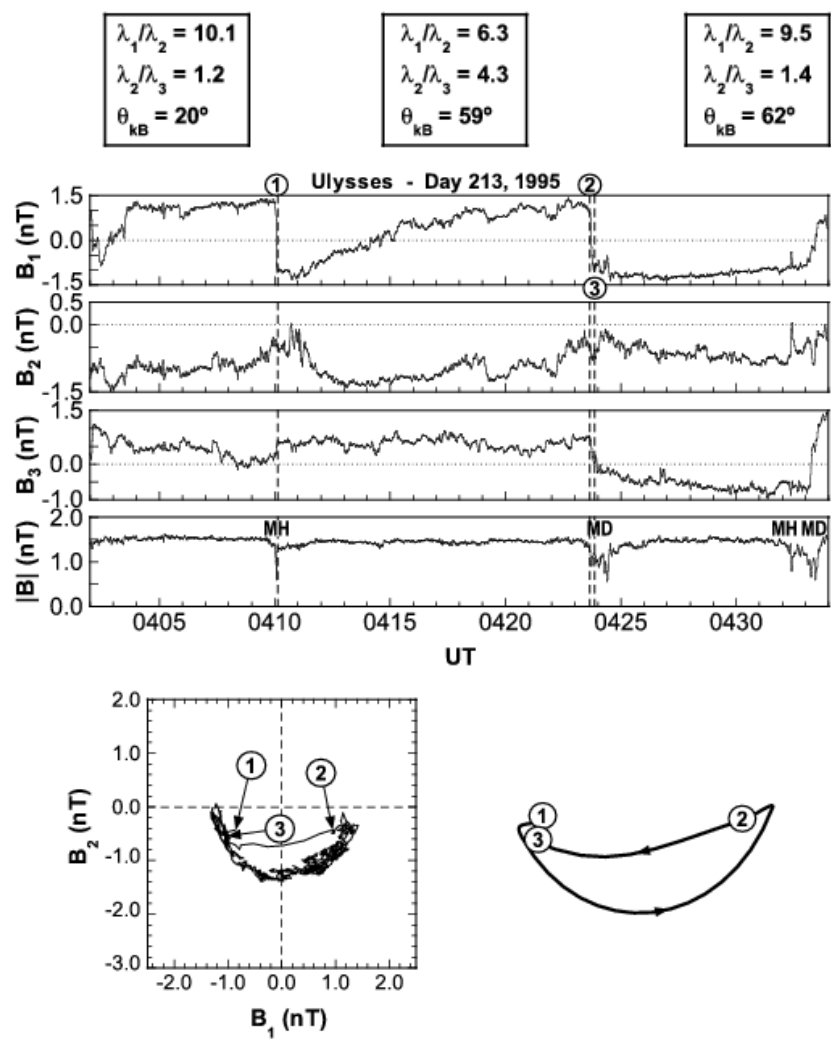

Fig. 3. The relationship between nonlinear Alfvén waves and MDs. The figure is taken from Tsurutani et al. (2002a). MDs/MHs are present at the phase-steepened edges of the Alfvén waves.

speed streams emanating from polar coronal holes, the solar wind propagates freely outward without obstruction. Thus the high $\beta$ regions are formed by the nonlinear processes of the wave itself.

Cases have been noted where the evolution of MDs and the Alfvén wave phase-steepened edges is rapid and detectable within $\sim 0.01 \mathrm{AU}$ distance (Tsurutani et al., 2005b). This evolution occurred in the ecliptic plane with the absence of any strong perturbing phenomena, such as fast magnetosonic shocks.

However closer to the ecliptic plane, MDs can be caused by another possible mechanism, shock-discontinuity interactions. This will be discussed below.

\subsubsection{MD formation associated with corotating interaction regions (CIRs)}

In the previous discussion, MDs were found in the radially flowing high speed solar wind emanating from a coronal hole (Fig. 1). A schematic showing a solar coronal hole is given in Fig. 4. A polar coronal hole is present at the north pole (shown in brown). The coronal hole is not symmetric about the pole and has a "finger" coming down to lower latitudes 


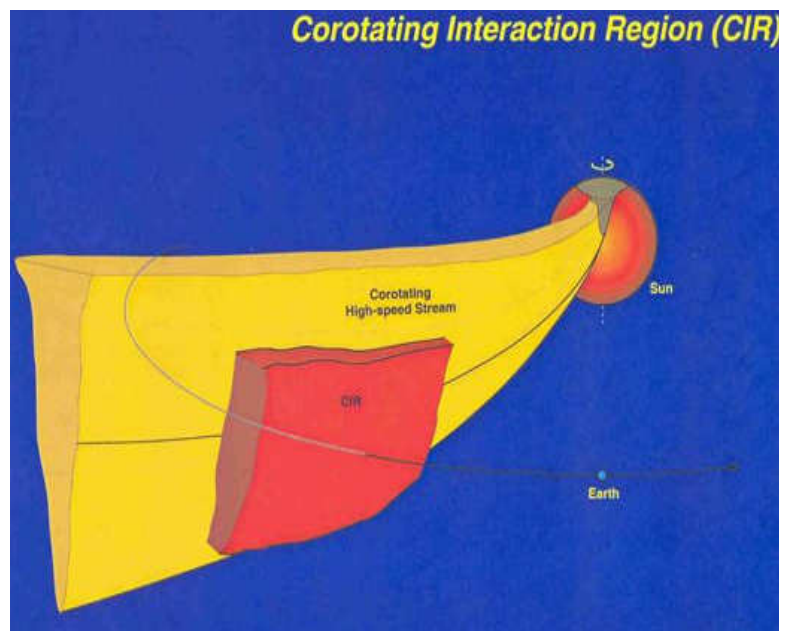

Fig. 4. A schematic showing how CIRs form at the leading edge of a high speed stream.

(facing the reader). If this finger persists for intervals longer than the solar rotation period of $\sim 27$ days, then an observer at the latitude of the finger will detect the high speed stream as the coronal hole faces it each solar rotation. The high speed stream has been said to "corotate" since the physical shape of the stream in interplanetary space is that of an Archimedean spiral. In actuality the high speed stream plasma and magnetic fields do not corotate, but the structure gives the appearance of doing so. A good analogy for this is a rotating water sprinkler. The profile of the water stream viewed from above has an Archimedean spiral shape.

Although the high speed stream from the polar region of the coronal hole expands radially outward into other high speed stream plasma, the solar wind coming out of the finger runs into solar wind plasma that is going at about half the speed (the slow solar wind). From the high speed solar wind-slow speed solar wind stream interaction, a plasma and field compression region is formed (Pizzo, 1985). This is indicated in the figure. The magnetically compressed region has been called a corotating interaction region or CIR (Smith and Wolf, 1976).

Figure 5 shows $\sim 5$ months of Ulysses field and plasma data when Ulysses was near the ecliptic plane. The solar wind velocity is shown in black (left-hand vertical scale) and the magnetic field magnitude in blue (far right-hand scale). MDs were identified in the high resolution magnetic field data by a computer program called the "interplanetary magnetic decrease automatic detection" (IMDAD, Guarnieri et al., 2009) code. A limit of $\Delta B_{0} / B_{\mathrm{L}}>0.5$ was chosen for the criterion for selecting a MD (the field decrease had to be $50 \%$ or greater than the larger ambient magnetic field on either side of the MD, $B_{\mathrm{L}}$ ). Further discussion can be found in Guarnieri et al. (2009). The plot in red is the number of MDs occurring in each calendar day and the scale is on the right.

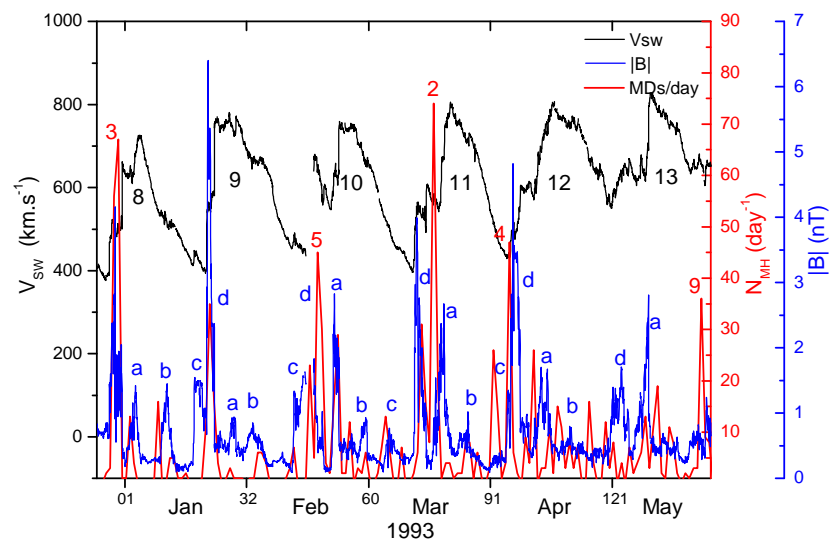

Fig. 5. The solar wind speed for six reappearances of a major high speed solar wind stream (listed as events 8 to 13), the magnetic field magnitude and the number of MDs/day. Although there is one major solar wind stream, there are several minor ones that lead to CIRs $a, b$ and $c$.

The streams recur about every $\sim 25$ days (the period is slightly different than stated before due to the lack of azimuthal rotation of Ulysses relative to the sun). The numbers indicate the 8th through the 13th reappearance of the high speed stream at Ulysses. Ulysses was traveling poleward at this time, so from the 11th through the 13th recurrence of the stream, Ulysses was becoming more and more embedded in the high stream proper. Thus the solar wind speed was becoming close to the constant high $750-800 \mathrm{~km} / \mathrm{s}$ value shown in Fig. 1 (over the pole).

The peak magnetic field regions (CIRs) are indicated by the labels a through $\mathrm{d}$. These are caused by the stream-stream interactions discussed previously. There are smaller streams in addition to the major ones, but discussion of this topic is complex and will not be pursued further.

What is most important for the present discussion is the large variability in MD occurrence rates. A very large peak (labeled "2") is noted in the middle of March 1993. Other peaks (3, 4 and 5) are noted as well. Peak 2 has a value of $77 \mathrm{MDs} /$ day. In sharp contrast, some days have no MDs at all. When a larger interval is examined (from 29 February 1992 to 14 September 1993), the average rate excluding the 10 largest peaks (thus this current numbering system) was $4.3 \pm 6.1 \mathrm{MDs} /$ day. The MD occurrence rate peaks noted in this figure are much greater than statistical fluctuations can explain.

Figure 6 shows the CIR associated with stream 8 in Fig. 5. From top to bottom, the panels are solar wind speed, proton density and temperature, the 3 components of the magnetic field, $B_{0}$, and the plasma $\beta$. The CIR is bounded by a forward magnetosonic (fast) shock labeled FS and a reverse magnetosonic (fast) shock labeled RS. Further discussion of the shock properties identified through the shock normal determination analyses and Rankine-Hugoniot conservation analyses can be found in Echer et al. (2010). At $\sim$ day 363.3, 


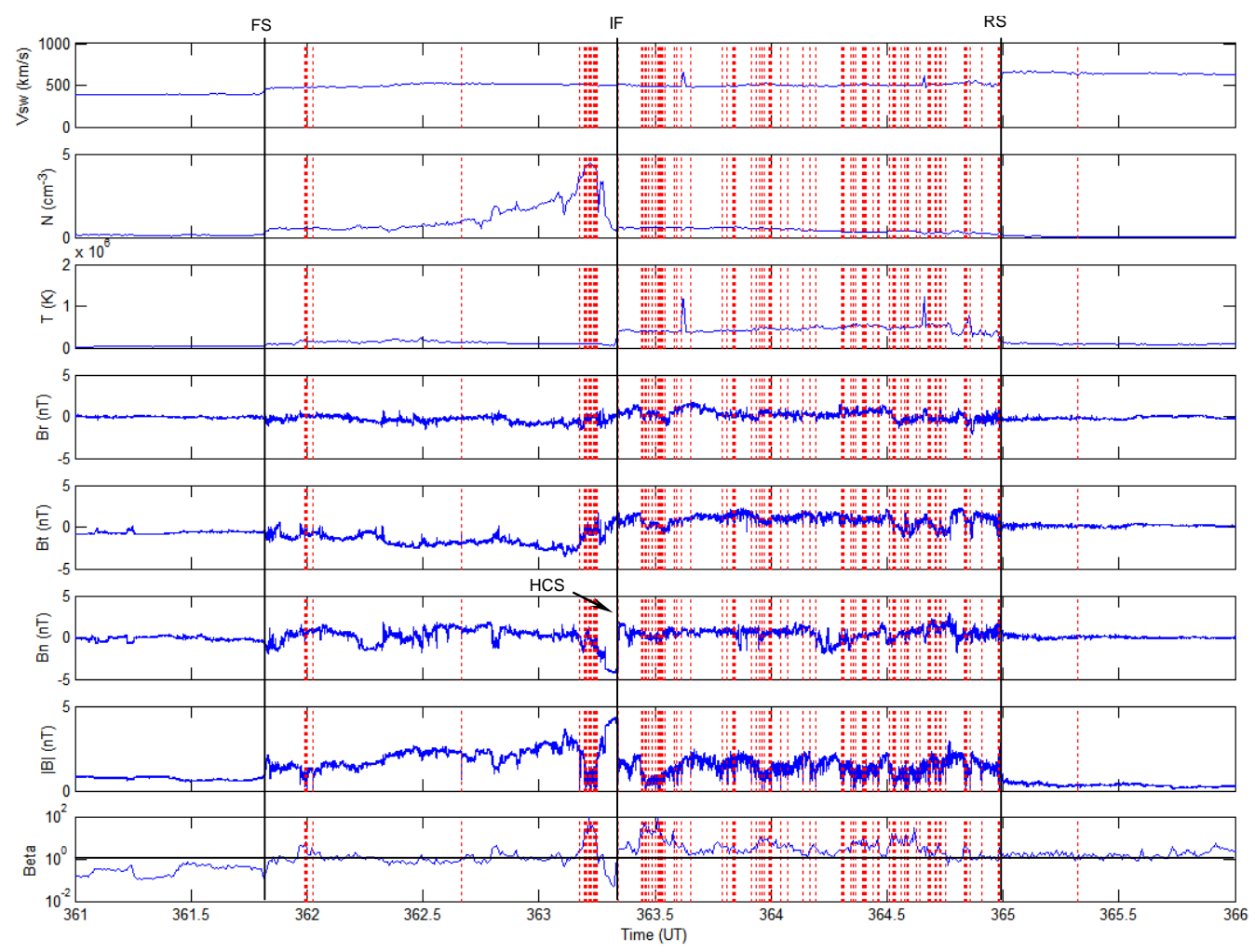

Fig. 6. CIR 8d shown in high resolution. The CIR is bounded by a fast forward shock (FS) and a fast reverse shock (RS). The interface (IF) is noted by a solid vertical line. Each MD identified by the IMDAD code is indicated by a dashed vertical line.

near the center of the CIR is a discontinuity that is believed to be the interface (IF) separating shocked and accelerated slow stream plasma (between the FS and the IF) from the shocked decelerated fast stream plasma (between the IF and the RS). This interface is believed to be formed when the high speed stream and slow speed stream first interact.

Individual MDs identified by the IMDAD code are indicated by the vertical dashed lines. The MD peak 3 of Fig. 6 is now resolved in higher resolution. Peak 3 was a double (two day duration) peak, consisting of 56 MDs for day 363 and 67 MDs for day 364, giving a total of 123 MDs for the two days.

There are several important features to note concerning where the MDs are located relative to the CIR. In the region both anti-sunward of the CIR (the interval between day 361 and 361.8) and sunward of the CIR (from day 365 to day 366) there is only one MD. The former region is the slow stream proper and the latter is the fast stream proper. Within the CIR itself, there is an asymmetry in the MD locations. Far more MDs are detected on the sunward side, between the IF and the RS. The final thing to note is that MDs occur where the plasma $\beta$ is high, from $1<\beta<10^{2}$. When $\beta<1$, there are no MDs. These features are typical of the MD-CIR relationship found in the study of the 15 high speed streams of the Ulysses near-ecliptic pass (Tsurutani et al., 2009).
There are MDs detected very close to the IF. A cluster of MDs is detected on $\sim$ day 363.2 and another on $\sim$ day 363.5. These parts of the CIR were formed close to the sun, $<1 \mathrm{AU}$. Tsurutani et al. (2009) have speculated that these are MDs that were formed close to the Sun and then convected outward by the solar wind. If this hypothesis is correct, these MDs are thus fossils that may give records of past plasma interactions. There are also MDs found close to the FS ( $\sim$ day 362.0$)$ and near the RS ( $\sim$ day 365.0). These parts of the CIR are newly formed plasma regions. It was therefore also concluded that these MDs were formed near the present location of the spacecraft, or at $\sim 5 \mathrm{AU}$.

\subsubsection{What is the mechanism forming high $\beta$ regions within CIRs?}

We first ask ourselves the question: "What causes the enormous variation in the plasma $\beta$ within the CIR?" And why is the $\beta$ significantly higher in the IF to RS region than in the FS to IF region? Our working hypothesis is shown in Fig. 7a and b. Figure 7a illustrates the spatial geometry of a CIR and the magnetic field lines lead to it. The antisolar portion is shaded in blue with the FS being furthest from the sun. The solar portion of the CIR is shaded pink with the RS closest to the sun. The IF separates the blue from the pink regions. The solar wind in the antisolar direction is the slow 
solar wind. Because the velocity is low, the Archimedean spiral magnetic field is wound up tighter. The high speed solar wind is in the region sunward of the CIR. Because of the higher speed there, the Archimedean spiral is more radial in this region than on the anti-sunward side.

The magnetic field direction as it contacts the CIR shocks defines the nature of the shock. In the case of the FS, the magnetic field is more tangential to its surface, so the shock is more quasiperpendicular (a perpendicular shock would have the field parallel to its surface). The RS is in general more quasiparallel than the FS.

Why is the shock quasiperpendicular/quasiparallel nature important for understanding plasma $\beta$ conditions? Let us discuss the extreme cases of a perpendicular and a parallel shock to illustrate the essential points. For a perpendicular shock, the magnetic field magnitude $B_{0}$ and plasma density $(N)$ are compressed. For a typical Mach 2-3 perpendicular CIR shock, both $B_{0}$ and $N$ increase by $\sim 2$ to 3 (Kennel et al., 1985). The ion and electron temperatures will increase primarily in the $T_{\perp}$ components. For a parallel shock, $N$ increases and $T_{\|}$increases, but there is no comparable change in $B_{0}$. Thus compression by a parallel shock increases the plasma $\beta$. If the shock is quasiparallel rather than parallel, then the plasma $\beta$ increase will be somewhat less than for the parallel case.

Figure $7 \mathrm{~b}$ is a more realistic case for CIR formation. The solar wind embedded magnetic field is not at a constant Archimedean spiral direction, but because of the presence of Alfvén waves and other stream-stream deflections, the field angles are in reality variable with time. These variable angles impinging on the FS and RS lead to variable plasma $\beta$ values as illustrated by the different shades of reds, pinks and blues (the redder the color, the higher the $\beta$ and the bluer the color, the lower the $\beta$ ).

\subsubsection{Mirror instability is not the mechanism for MD formation in CIRs}

Because of: 1) the quasiparallel nature of the RS and the formation of MDs in the sunward side of CIRs and 2) the quasiperpendicular nature of the FS and the lack of MDs in the antisunward side of CIRs, Tsurutani et al. (2009) concluded that the mirror mode instability was an unlikely mechanism for MD formation at CIRs. Plasma heating at quasiparallel shocks (RSs) would lead to large $\beta_{\|} / \beta_{\perp}>1$ anisotropies, a situation that would lead to mirror stability rather than instability. Other suggested mechanisms for MD formation at CIRs are: 1) plasma interactions with rotational discontinuities downstream of the shock (Tsubouchi and Matsumoto, 2005), 2) wave-wave interactions in the sheath (Vasquez et al., 2007; Tsubouchi, 2009), 3) nonlinear evolution of large-amplitude right-hand polarized obliquely propagating Alfvén wave packets (Buti et al., 2001), and 4) compression of phase-steepened Alfvén wave by the RS or propagation into high $\beta$ plasma regions (Tsurutani et al., (a)

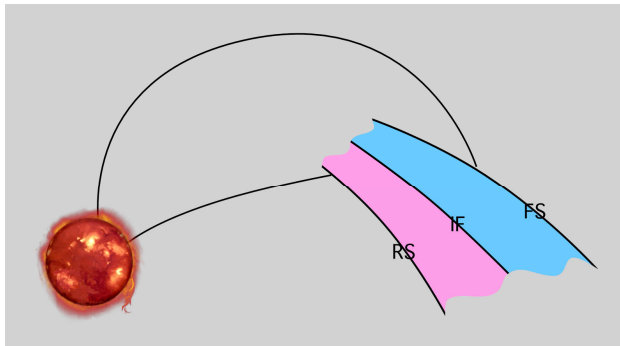

(b)

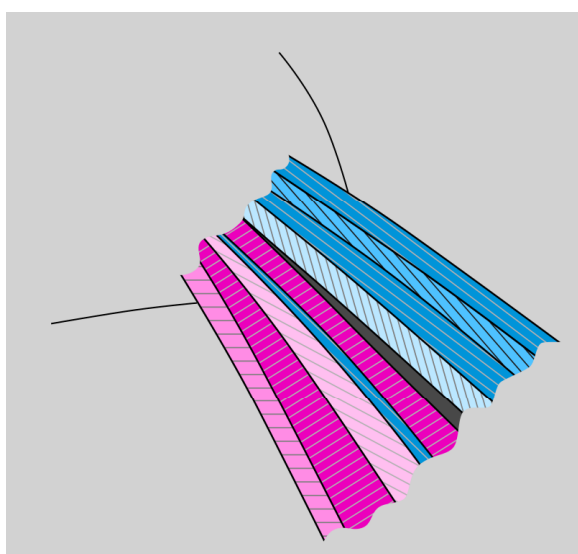

Fig. 7. Schematics showing the geometry of a CIR, the upstream and downstream interplanetary magnetic fields and the forward and reverse shocks (panel a). Panel $\mathbf{b}$ shows a more realistic plasma beta distribution of the CIR. This is due to variations of the upstream and downstream magnetic field orientations.

2002a, b). It is clear that many of these different ideas are overlapping in concept.

\subsubsection{Statistical properties of Ulysses MDs}

Figure 8 shows the radial direction thickness distribution of MDs identified in the Ulysses ecliptic pass. A total of 3920 events were identified. The thickness was determined by using the measured solar wind speed and the time duration of the MD crossing. The tail of the distribution is shown as an insert. An exponential fit to the distribution was made in Tsurutani et al. (2009) with $N=A_{1} e^{-\left(t / t_{1}\right)}$, where $A_{1}=2173 \pm 35$ and $t_{1}=173 \mathrm{~s}$. The general result of a decreasing number of MDs with increasing thicknesses is consistent with the results of previous studies (Tsurutani and Ho, 1999; Stevens and Kasper, 2007).

Figure 9 shows the distribution of angular changes across MDs. A fit gives $\% \mathrm{MD}=2+48 e^{-\left(\Delta \theta^{\circ} / 18.8^{\circ}\right)}$ (not shown for brevity) where $\Delta \theta$ is the angular change. MDs have a broad range of angular changes occurring across them. Small angular changes occur most frequently, but changes with $\Delta \theta=170^{\circ}$ to $180^{\circ}$ are present.

"Linear" MDs are defined as those with $\Delta \theta \leq 10^{\circ}$ (Burlaga and Lemaire, 1978; Winterhalter et al., 1994, 1995). 


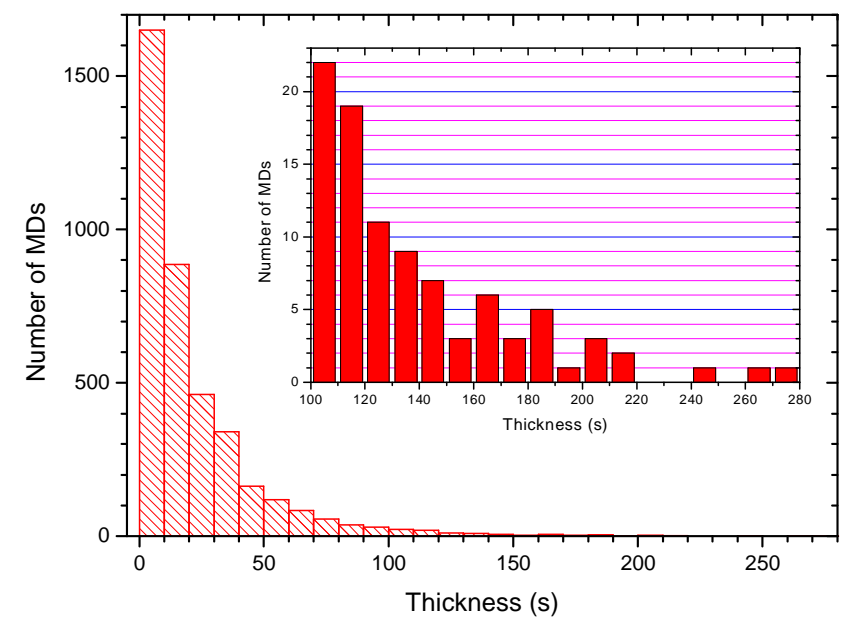

Fig. 8. The (temporal) "thickness" of MDs. The minimum thickness is $2-3 \rho_{p}$. The maximum thickness extends to hundreds of $\rho_{p}$.

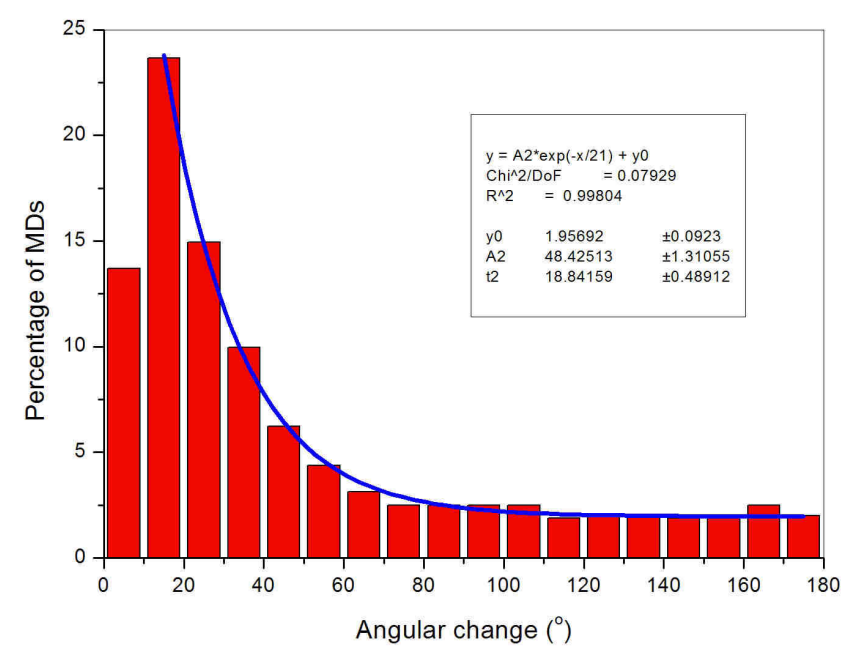

Fig. 9. The magnetic field angular change from one MD to an adjacent one. All angular changes are detected.

In our study we find that only $13.5 \%$ of MDs are "linear". This is in good agreement with the findings of Winterhalter et al. (1994). They are a small percentage of MDs and perhaps merit no special attention from researchers.

\subsection{Mirror mode structures}

As previously mentioned, mirror mode structures are generated by an instability associated with an anisotropic plasma such that $\beta_{\perp} / \beta_{\|}>1+1 / \beta_{\perp}$. It is clear from this expression that the higher the $\beta$ of the ambient plasma, the more easily this instability will take place. Mirror mode structures were first identified in the magnetosheaths of the Earth, Jupiter and Saturn. Examples will be shown to compare and contrast them to MDs.
Figure 10 shows the first observation of mirror modes in the Earth's magnetosheath taken by ISEE-1 instrumentation. The structures were quasiperiodic, with a mean scale of $\sim 20 \rho_{\mathrm{p}}$ separating adjacent structures, and had little or no angular changes between the structures. The unit $\rho_{\mathrm{p}}$ is the local proton gyroradius.

Figure 11 illustrates the relationship between the plasma and magnetic field pressures for several cycles of a mirror mode structure. It is noted that the plasma pressure is $\sim 180^{\circ}$ out-of-phase with the magnetic pressure and the total pressure is constant, to the first order. The mirror instability creates regions of alternating high $\beta$ (magnetic field dips) and low $\beta$ regions.

Figure 12 displays the same mirror mode structures detected at two spacecraft within the Earth's magnetosheath (ISEE-1 and -2). The magnetosheath plasma convective time shift has been calculated from the measures plasma speed and direction and the delay is indicated by shift in the two plots. What is apparent is that the mirror mode structures are first detected by the ISEE-2 instrument and then by the ISEE-1 instrument. Since the structures are close to identical in the two spacecraft, the mirror mode structures are essentially convected by the magnetosheath plasma. This is in agreement with the predictions of Hasegawa $(1969,1975)$ and the modeling results of Price et al. (1986).

Figure 13 shows the entire Pioneer 11 pass through the magnetosheath of Saturn. The bow shock is encountered at $\sim 18: 10$ UT, the magnetopause at $\sim 22: 00$ UT and the magnetosphere proper after 22:00 UT. Mirror mode structures start to form at about 19:00 UT and reach their largest amplitudes close to the magnetopause. This is typical of the location of mirror modes in all planetary magnetosheaths observed to date. There is little or no structure near the bow shock and the greatest amplitudes are detected near the magnetopause.

\subsubsection{Sources of free energy for mirror instability in planetary magnetosheaths}

There are several possible mechanisms that can lead to large $\beta_{\perp} / \beta_{\|}>1$. One is magnetic field line draping around the planetary magnetopause (Midgley and Davis, 1963; Zwan and Wolf, 1976). Draping leads to squeezing of the hot $T_{||}$plasma along the lines of force into the downstream region. Also the magnetic tension near the local noon nose leads to increase in $T_{\perp}$ in this region. A second mechanism is plasma and magnetic compression as the solar wind crosses the quasiperpendicular portion of the bow shock. As discussed previously, heating at the perpendicular portion of the bow shock will be the primary region that leads to anisotropies that are conducive for mirror stability. Plasma heating across the quasiparallel portion of the bow shock will give plasma anisotropies that stabilize the mirror mode.

We have discussed several important observations pertaining to mirror modes in planetary magnetosheaths: 1) mirror mode structures are rarely if ever detected immediately 
ISEE 1

20 November 1977

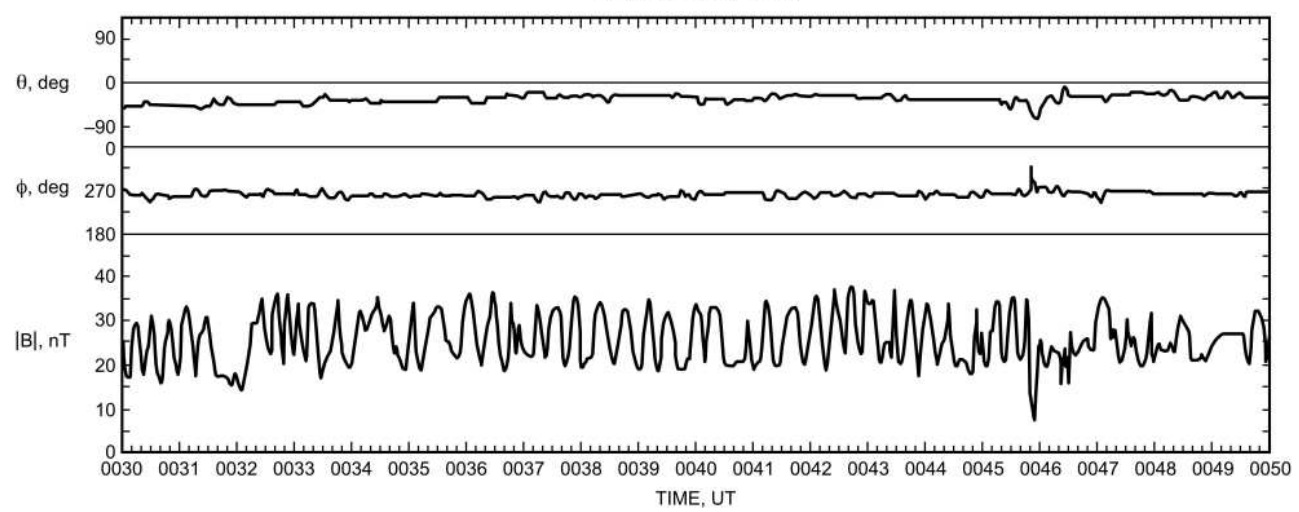

Fig. 10. Mirror mode structures in the Earth's magnetosheath. The structures are quasiperiodic and there are little or no field directional changes across them.

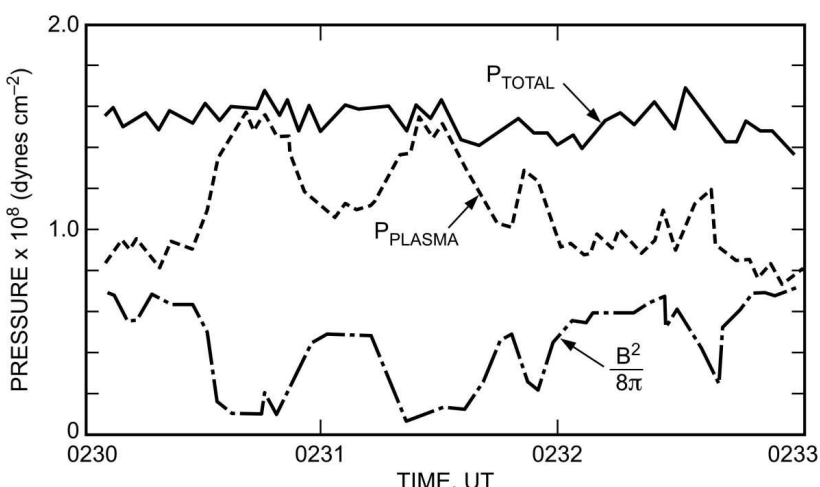

Fig. 11. The plasma and magnetic field partial pressures and the total (plasma plus magnetic field pressure) for several cycles of mirror mode structures.

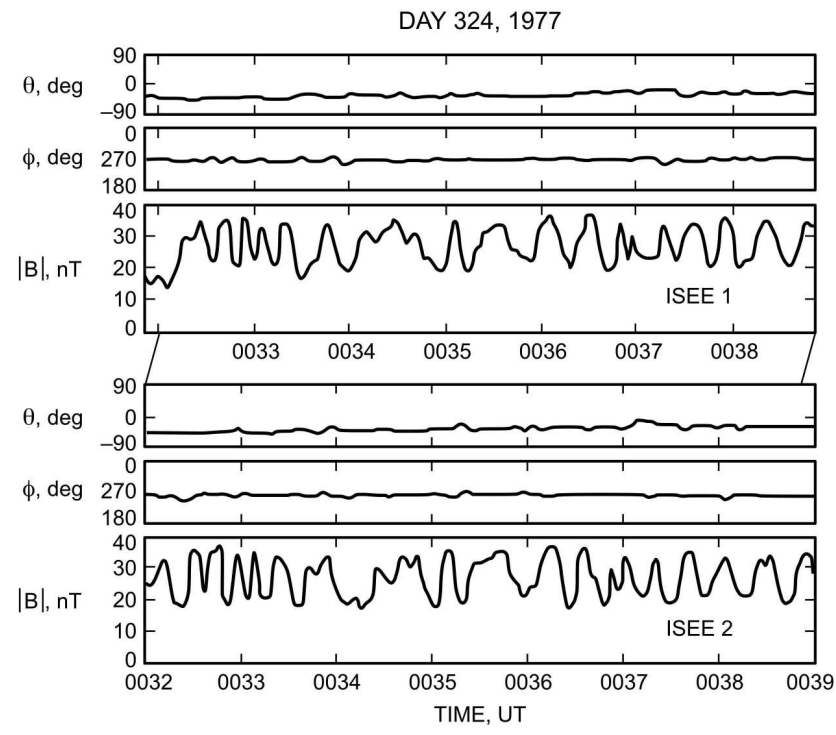

Fig. 12. Mirror mode structures detected by two closely separated spacecraft (ISEE-1 and -2). The magnetosheath flow convects the structures from ISEE-2 to ISEE-1.
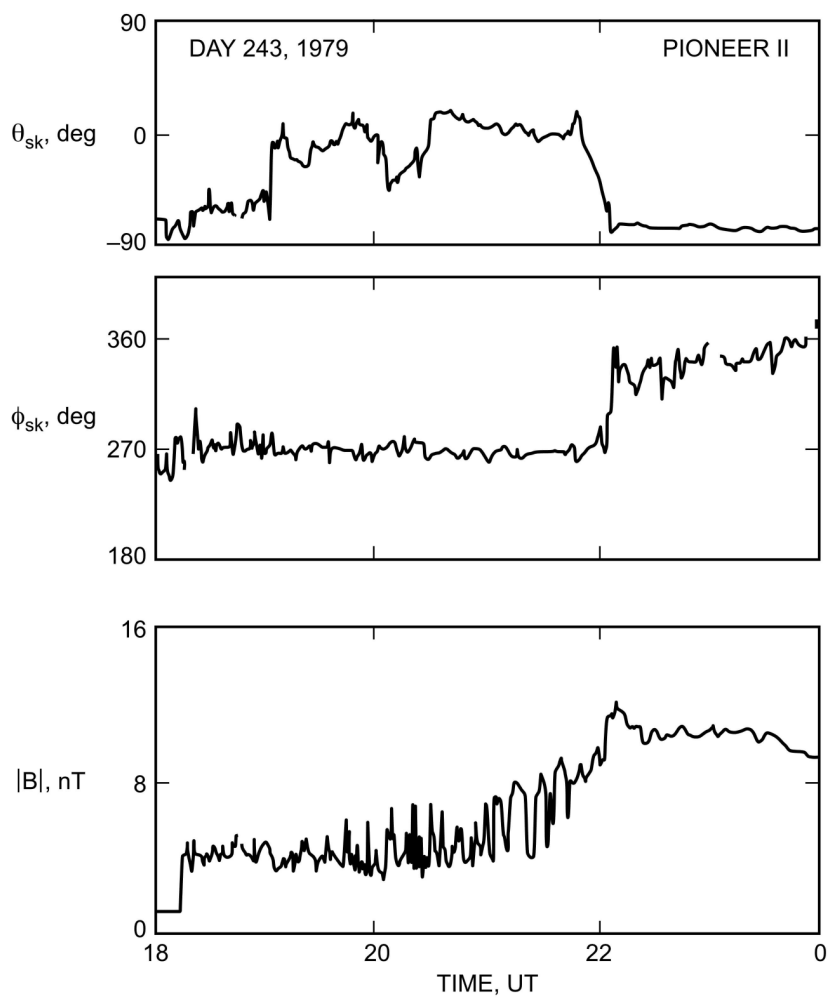

Fig. 13. Mirror mode structures in Saturn's magnetosheath. Note that the amplitudes are small or nonexistent near the shock and largest near the magnetopause.

downstream of quasiperpendicular/perpendicular shocks, 2) MDs are rarely detected downstream of CIR forward shocks and 3) the mirror mode structures have the largest amplitudes closest to the magnetopause. From this we conclude that both sources of free energy are important. There is no doubt that quasiperpendicular shock compression will heat the ions in $T_{\perp}$. Magnetic field line draping is also certainly playing an 
important role. The latter can be concluded by observation $3)$. The relative contribution of each may depend on the interplanetary conditions (plasma $\beta$, solar wind Mach number, magnetic field Archimedean spiral angle, etc.). Further research is needed on this topic.

\subsubsection{The ion cyclotron instability}

One of the biggest theoretical puzzles of mirror mode structures in planetary magnetosheaths is why they are present at all? Theoretical calculations show that the ion cyclotron instability linear growth rate (Kennel and Petschek, 1966) in an electron-proton plasma is higher than that for the mirror instability. Gary (1992) have added helium ions into the solar wind plasma in their calculations to create stop bands in the ion cyclotron dispersion relationship. These stop bands effectively reduce the linear growth rate of the ion cyclotron waves to values below the mirror mode linear growth rates. Brinca and Tsurutani (1988) have shown that these additional ions do not affect the linear mirror mode growth rates. Gary (1992) also noted that the low $\beta$ plasma conditions also led to higher linear ion cyclotron growth compared to the mirror instability. Another approach has been taken by Shoji et al. (2009). They have used a 3-D hybrid code to show that because of the larger phase space volume taken by the obliquely propagating mirror mode structures, this instability can use up the free energy rapidly, effectively leading to a cessation of the ion cyclotron wave growth. This topic is still being debated and explored at this time.

\subsection{MDS and mirror modes: How to distinguish them?}

We have shown that MDs and mirror modes are observationally quite different structures. They also occur in different regions of space, MDs in interplanetary space and mirror modes in planetary magnetosheaths. MDs are characterized by: 1) variable (exponentially decreasing) angular changes, 2) variable (exponentially decreasing) thicknesses, and 3) no obvious inter-event spacings. In sharp contrast, mirror modes are characterized by: 1) little or no angular changes, 2) having a characteristic scale size, and 3) are quasiperiodic in nature.

\subsubsection{The heliosheath}

Dips in the heliosheath magnetic field have recently been detected by the Voyager spacecraft (Burlaga et al., 2006, 2007). What are these structures? Liu et al. (2007) have suggested that perpendicular shock compression of upstream plasma can produce mirror instability at the termination shock.

Figure 14 gives the polar angular changes of the magnetic field across the magnetic dips observed by Voyager 1 at $\sim 95 \mathrm{AU}$ from the sun (Tsurutani et al., 2010). It is noted that the changes in both $\theta$ and $\phi$ are small. The mean change

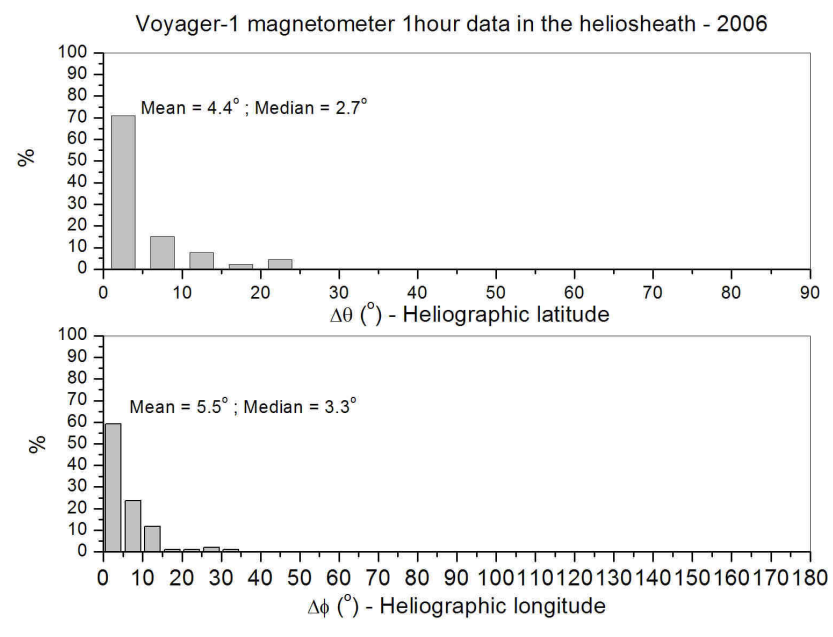

Fig. 14. The angular changes of the magnetic field from magnetic dip to dip in the heliosheath. The angular changes are quite small.

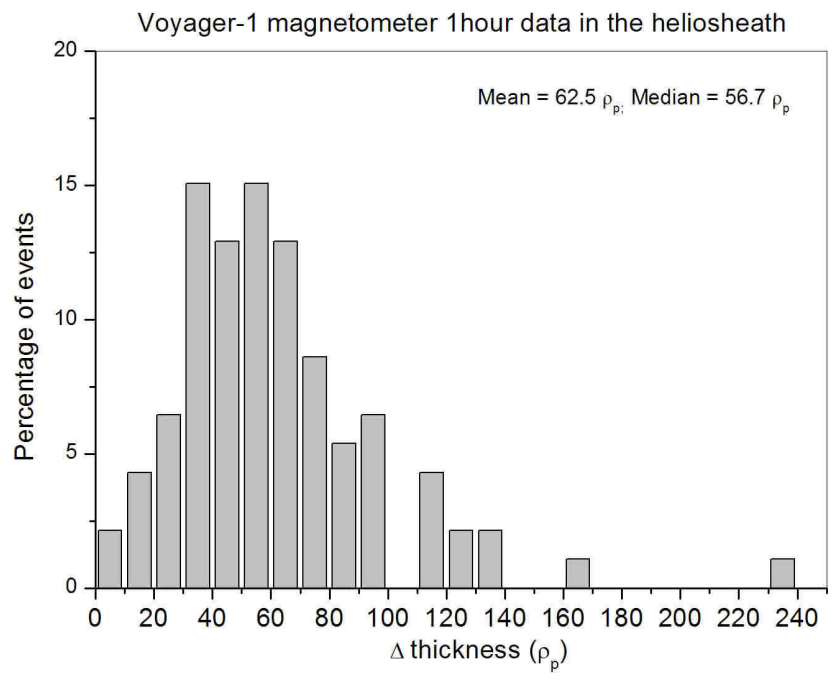

Fig. 15. The spacings between adjacent magnetic dips in the heliosheath.

in $\theta$ is $4.4^{\circ}$ and in $\phi$ is $5.5^{\circ}$. It should be noted that no cases of large angular changes were noted in either the $\theta$ or $\phi$ distributions. These features are consistent with the heliosheath field dips being mirror mode structures.

Figure 15 shows the inter-dip spacings. There is a wide range spanning from $0-10 \rho_{\mathrm{p}}$ to as large as $230-240 \rho_{\mathrm{p}}$. However there is clear a peak in the distribution. This occurs at $62.5 \rho_{\mathrm{p}}$. This distribution is unlike that of MDs and is more similar to mirror mode separations. 


\subsubsection{If mirror mode instability is the cause of the heliosheath dips, what are the sources of free energy?}

The interplanetary conditions at very large distances from the sun are much different from those near $1 \mathrm{AU}$. The typical magnetic field Archimedean spiral angle should be close to $90^{\circ}$ in comparison to a $45^{\circ}$ angle near the Earth. Secondly, interstellar neutrals will play an important role at these large distances. As the neutrals enter the heliosphere, they will become ionized by charge exchange. As the neutral hydrogen atoms are changed into protons, they are "picked up" by the convected interplanetary magnetic field (an electric field in the frame of the proton) and will "mass load" the solar wind. The pickup of interstellar neutrals will be occurring throughout interplanetary space. However the greatest amount of ion pickup will occur close to the boundary where the neutrals enter the heliosphere, near the termination shock and within the heliosheath. The additional mass will cause a deceleration which has been measured. Richardson (2008) and Richardson and Stone (2008) have noted a 20-30\% decrease in solar wind speed as Voyager 2 approached the heliopause. For purposes of calculation, a conservative deceleration of $20 \%$ was used. This implies a $25 \%$ increase in the solar wind mass.

As the ions become "picked up" by the solar wind, they will constitute either a beam (if the magnetic field is parallel to the solar wind flow) or a ring (if the magnetic field is orthogonal to the solar wind flow (Wu and Davidson, 1972; Tsurutani and Smith, 1986a, b, 1997). Different magnetic field orientations lead to different plasma instabilities (Brinca and Tsurutani, 1987, 1988, 1989). Because of the tightly wound Archimedean spiral magnetic fields at distances near the heliopause, the magnetic fields should be orthogonal to the solar wind flow and the pickup ions will form rings. The rings will constitute $\beta_{\perp} / \beta_{\|}>1$ anisotropies which will contribute to the free energy for either mirror mode or ion cyclotron wave instabilities. If we assume upstream solar wind values of $N=10^{-3} \mathrm{~cm}^{-3}, B_{0}=5 \times 10^{-2} \mathrm{nT}$ and $T=10^{4} \mathrm{~K}$, one get a $\beta=0.14$. The rest of the calculation follows easily. The pickup ion density will be $N_{\mathrm{p}}=2.5 \times 10^{-4} \mathrm{~cm}^{-3}$. The pickup ion perpendicular kinetic energy will be $\sim 1 \mathrm{keV}$. Thus the plasma $\beta$ due to the pickup ions alone will be $\beta_{\mathrm{p}}=40$. If the magnetic field is not purely orthogonal to the solar wind flow direction but varies by $\sim 10^{\circ}$ relative to the presence of Alfvén waves, then $\beta_{\perp} / \beta_{\|} \sim 9$, much larger than the instability criteria of $\sim 1$.

If the upstream magnetic field is nearly orthogonal to the solar wind flow in the upstream region, then the field will also be nearly parallel to the termination shock. The shock will thus be almost perpendicular in nature. Additional heating of the plasma crossing the shock will occur in the perpendicular to the field $\left(T_{\perp}\right)$ direction. This will be an additional source for mirror instability.
A third source of free energy is the pickup of interstellar neutrals in the heliosheath. The flow speed is lower so the perpendicular kinetic energy pickup per particle will be less than in the upstream region. Modeling should help identify the relative importance of these various sources. The biggest unknown is the determination of the branching of free energy towards ion cyclotron waves and mirror mode structures. As long as this topic is under debate, the issue of the importance of various sources of free energy will remain unclear.

\section{Conclusions}

A brief review of observations of MDs in interplanetary space and mirror modes in planetary magnetosheaths and the heliosheath have been provided so that the reader should be able to distinguish these different types of "magnetic dips" from observations. The potential physical mechanisms leading to their generation have been discussed with main references included so that interested researchers may contribute to further understanding of these important physical processes. Arguments have been presented that indicate that the heliosheath dips are indeed mirror mode structures and the sources of free energy are threefold: interstellar ion pickup in the upstream region, perpendicular-to-the field heating at the quasiperpendicular termination shock, and interstellar ion pickup in the heliosheath. The evolution of the mirror mode structures as Voyager penetrates deeper into the heliosheath should be of great interest to the readers.

The creation of high $\beta$ regions by nonlinear processes should be of interest to astrophysical researchers. In particular the propagation of energetic particles through the anisotropic plasma will be influenced strongly (Tsurutani et al., 1999; Tsurutani and Lakhina, 2004; Costa et al., 2010).

Acknowledgements. Portions of this work were performed at the Jet Propulsion Laboratory, California Institute of Technology under contract with NASA. EE would like to acknowledge support from FAPESP contract 2007/5233-1 and CNPq contract 300211/2008-2. GSL thanks the Indian National Science Academy New Delhi for support under the Senior Science Scheme. BTT would like to thank the Tech. Univ. Braunschweig for their kind support during his sabbatical leave at that institution.

Edited by: G. Morales

Reviewed by: S. P. Gary and another anonymous referee

\section{References}

Balogh, A., Smith, E. J., Tsurutani, B. T., Southwood, D. J., Forsyth, R. J., and Horbury, T. S.: The heliospheric magnetic field over the south polar region of the sun, Science, 268, p. 1997, 1995.

Belcher, J. W. and Davis Jr., L.: Large-amplitude Alfvén waves in the interplanetary medium: 2, J. Geophys. Res., 76, p. 3534, 1971. 
Brinca, A. L. and Tsurutani, B. T.: Survey of low frequency electromagnetic waves stimulated by two coexisting newborn ion species, J. Geophys. Res., 93, p. 48, 1988.

Brinca, A. L. and Tsurutani, B. T.: On the polarization, compression and nonoscillatory behavior of hydromagnetic waves associated with pickup ions, Geophys. Res. Lett., 14, p. 495, 1987.

Brinca, A. L. and Tsurutani, B. T.: Survey of low-frequency electromagnetic waves stimulated by two coexisting newborn ion species, J. Geophys. Res., 93, p. 48, 1988.

Brinca, A. L. and Tsurutani, B. T.: Influence of multiple ion species on Low-Frequency electromagnetic wave instabilities, J. Geophys. Res., 94(A10), p. 13565, 1989.

Brinca, A. L. and Tsurutani, B. T.: On the excitation of cyclotron harmonic waves by newborn heavy ions, J. Geophys. Res., 94, p. 5467, 1989.

Burlaga, L. F. and Lemaire, J. F.: Interplanetary magnetic holes: Theory, J. Geophys. Res., 83, p. 5157, 1978.

Burlaga, L. F., Ness, N. F., and Acuna, M. H.: Magnetic fields in the heliosheath: Voyager 1 observations, Astrophys. J., 642, p. 584, 2006.

Burlaga, L. F., Ness, N. F., and Acuna, M. H.: Linear magnetic holes in a unipolar region of the heliosheath observed by Voyager 1, J. Geophys. Res., 112, A07106, doi:10.1029/2007JA012292, 2007.

Buti, B., Tsurutani, B. T., Neugebauer, M., and Goldstein, B. E.: Generation mechanism for magnetic holes in the solar wind, Geophys. Res. Lett., 28, 1355-1358, 2001.

Chandrasekhar, S. A., Kaufman, A. N., and Watson, K. M.: The stability of the pinch, Proc. R. Soc. Lon. Ser.-A., 245, p. 435, 1958.

Costa, E. Jr., E. Echer, M.V. Alves. B.T. Tsurutani, F.J.R. Simoes Jr., F.R. Cardoso and G.S. Lakhina, A computational study of nonresonant cross-field diffusion of energetic particles due to their interaction with interplanetary magnetic decreases, J. Atmos. Sol.-Terr. Phys., submitted, 2010.

Dasgupta, B., Tsurutani, B. T., and Janaki, M. S.: A kinetic approach to the Pondermotive Force, Geophys. Res. Lett., 30, SSC 11-1, doi:10.1029/2003GL017385, 2003.

Echer, E., Tsurutani, B. T., and Guarnieri, F. L.: Forward and reverse CIR shocks at 4-5 AU: Ulysses, Adv. Space Res., 45, p. 798, 2010.

Fränz, M., Burgess, D., and Horbury, T. S.: Magnetic depressions in the solar wind, J. Geophys. Res., 105, p. 12725, 2000.

Gary, S. P.: The mirror and ion cyclotron anisotropy instabilities, J. Geophys. Res., 97, p. 8519, 1992.

Guarnieri, F. L., Tsurutani, B. T., and Echer, E.: The interplanetary magnetic decrease automatic detection (IMDAD) code, Earth Planets Space, 61, 585, 2009

Hada, T.: Evolution of large-amplitude Alfvén waves in solar wind, Geophys. Res. Lett., 20, p. 2415, 1993.

Hasegawa, A.: Drift mirror instability in the magnetosphere, Phys. Fluids, 12, p. 2642, 1969.

Hasegawa, A.: Plasma Instabilities and Nonlinear Effects, in: Physics and Chemistry in Space 8, p. 94, Springer-Verlag, New York, 1975.

Kennel, C. F. and Petschek, H. E.: Limit of stably trapped particle fluxes, J. Geophys. Res., 71, p. 1, 1966.

Kennel, C. F., Edmiston, J. P., and Hada, T.: A quarter century of collisionless shock research, in: Collisionless Shocks in the
Heliosphere: A Tutorial Review, edited by: Stone, R. G. and Tsurutani, B. T., AGU Monograph, 34, p. 1, 1985.

Medvedev, M. V., Diamond, P. H., Shevchenko, V. I., and Galinsky, V. L.: Dissipative dynamics of collisionless nonlinear Alfvén wave trainss, Phys. Rev. Lett., 78, p. 4934, 1997.

Midgley, J. E. and Davis Jr., L.: Calculation by a moment technique of the perturbation of the geomagnetic field by the solar wind, J. Geophys. Res., 68, p. 5111, 1963.

Phillips, J. L., Bame, S. J., Feldman, W. C., Goldstein, B. E., Gosling, J. T., Hammond, C. M., McComas, D. J., Neugebauer, M., Scime, E. E., and Suess, S. T.: Ulysses solar wind plasma observations at high southerly latitudes, Science, 268, p. 1030, 1995.

Pizzo, V. J.: Interplanetary shocks on the large scale: A retrospective on the last decade's theoretical efforts, in: Collisionless Shocks in the Heliosphere: Reviews of Current Research, edited by: Tsurutani, B. T. and Stone, R. G., AGU Press, Washington DC, 35, p. 51, 1985.

Pokhotelov, O. A., Sagdeev, R. Z., Balikhin, M. A., and Treumann, R. A.: Mirror instability at finite ion-Larmor radius wavelengths, J. Geophys. Res., 109, A09213, doi:10.1029/2004JA010568, 2004.

Price, C. P., Swift, D. W., and Lee, L.-C.: Numerical simulation of nonoscillatory mirror mode waves at the Earth's magnetosheath, J. Geophys. Res., 91, p. 101, 1986.

Richardson, J. D.: Plasma near the termination shock and the heliosheath, in: CP1029, Particle Acceleration and Transport in the Heliosphere and Beyond..., 7th Annual Astrophysics Conference, edited by: Li, G., Verkhoglyadova, O., Zank, G. D., et al., Amer. Inst. Phys, NY, 2008.

Richardson, J. D. and Stone, E. C.: The solar wind in the outer heliosphere, Space Sci. Rev., 143, doi:10.1007/s11214-008-9443-z, 2008.

Shoji, M., Omura, Y., Tsurutani, B. T., Verkhoglyadova, O. P., and Lembege, B.: Mirror instability and L-mode electromagnetic ion cyclotron instability: Competition in the Earth's magnetosphere, J. Geophys. Res., 114, A10203, doi:10.1029/2008JA014038, 2009.

Smith, E. J. and Wolfe, J. H.: Observations of interaction regions and corotating shocks between one and five AU: Pioneers 10 and 11, Geophys. Res. Lett., 3, p. 137, 1976.

Southwood, D. J. and Kivelson, M. G.: Mirror instability: 1. Physical mechanism of linear instability, J. Geophys. Res., 98, p. 9181, 1993.

Stevens, M. L. and Kasper, J. C.: A scale-free analysis of magnetic holes at $1 \mathrm{AU}$, J. Geophys. Res., 112, A05109, doi:10.1029/2006JA012116, 2007.

Tsubouchi, K. and Matsumoto, H.: Effect of upstream rotational field on the formation of magnetic depressions in a quasi-perpendicular shock downstream, J. Geophys. Res., 110, A04101, doi:10.1029/2004JA010818, 2005.

Tsubouchi, K.: Alfvén wave evolution within corotating interaction regions associated with the formation of magnetic holes/decreases, J. Geophys. Res., 114, A02101, doi:10.1029/2008JA013568, 2009.

Tsurutani, B. T., Smith, E. J., Anderson, R. R., Ogilvie, K. W., Scudder, J. D., Baker, D. N., and Bame, S. J.: Lion roars and nonoscillatory drift mirror waves in the magnetosheath, J. Geophys. Res., 87, p. 6060, 1982. 
Tsurutani, B. T. and Smith, E. J.: Strong hydromagnetic turbulence associated with comet Giacobini-Zinner, Geophys. Res. Lett., 13, p. 259, 1986a.

Tsurutani, B. T. and Smith, E. J.: Hydromagnetic waves and instabilities associated with cometary ion pickup: ICE observations, Geophys. Res. Lett., 13, 263, 1986 b.

Tsurutani, B. T., Ho, C. M., Smith, E. J., Neugebauer, M., Goldstein, B. E., Mok, J. S., Arballo, J. K., Balogh, A., Southwood, D. J., and Feldman, W. C.: The relationship between interplanetary discontinuities and Alfvén waves: Ulysses observations, Geophys. Res., Lett., 21, p. 2267, 1994.

Tsurutani, B. T., Glassmeier, K.-H., and Neugebauer, F. M.: A review of nonlinear low frequency (LF) wave observations in space plasma: On the development of plasma turbulence, in: Nonlinear Waves and Chaos in Space Plasmas, edited by: Hada, T. and Matsumoto, H., Terra Sci. Publ. Co, Tokyo, p. 1, 1997.

Tsurutani, B. T. and Ho, C. M.: A review of discontinuities and Alfvén waves in interplanetary space: Ulysses results, Rev. Geophys., 37, p. 517, 1999.

Tsurutani, B. T. and Lakhina, G. S.: Cross-field particle diffusion in a collisionless plasma: A nonresonant and a resonant mechanism, in CP 703, in: Plasmas in the laboratory and in the universe, AIP Publs., Ney York, 2004.

Tsurutani, B. T., Lakhina, G. S., Winterhalter, D., Arballo, J. K., Galvan, C., and Sukurai, R.: Energetic particle cross-field diffusion: Interaction with Magnetic Decreases (MDs), Nonlin. Processes Geophys., 6, 235-242, doi:10.5194/npg-6-235-1999, 1999.

Tsurutani, B. T., Dasgupta, B., Galvan, C., Neugebauer, M., Lakhina, G. S., Arballo, J. K., Winterhalter, D., Goldstein, B. E., and Buti, B.: Phase-steepened Alfvén waves, proton perpendicular energization and creation of magnetic holes and magnetic decreases: The ponderomotive force, Geophys. Res. Lett., 29(24), 2233, doi:10.1029/2002GL015652, 2002a.

Tsurutani, B. T., Galvan, C., Arballo, J. K., Winterhalter, D., Sakurai, R., Smith, E. J., Buti, B., Lakhina, G. S., and Balogh, A.: Relationship between discontinuities, magnetic holes, magnetic decreases, and nonlinear Alfvén waves: Ulysses observations over the solar poles, Geophys. Res. Lett., 29(11), 1528, doi:10.1029/2001GL013623, 2002b.

Tsurutani, B. T., Lakhina, G. S., Pickett, J. S., Guarnieri, F. L., Lin, N., and Goldstein, B. E.: Nonlinear Alfvén waves, discontinuities, proton perpendicular acceleration, and magnetic holes/decreases in interplanetary space and the magnetosphere: intermediate shocks?, Nonlin. Processes Geophys., 12, 321-336, doi:10.5194/npg-12-321-2005, 2005a.
Tsurutani, B. T., Guarnieri, F. L., Lakhina, G. S., and Hada, T.: Rapid evolution of magnetic decreases (MDs) and discontinuities in the solar wind: ACE and CLUSTER, Geophys. Res. Lett., 32, L10103, doi:10.1029/2004GL022151, 2005b.

Tsurutani, B. T., Guarneiri, F. L., Echer, E., Lakhina, G. S., and Verkhoglyadova, O. P.: Magnetic decrease (MD) formation from $<1 \mathrm{AU}$ to $\sim 5 \mathrm{AU}$ : Corotating interaction region reverse shocks, J. Geophys. Res., 114, A08105, doi:10.1029/2008JA013927, 2009.

Tsurutani, B. T., Echer, E., Verkhoglyadova, O. P., and Lakhina, G. S.: Mirror instability upstream of the termination shock (TS) and in the heliosheath, J. Atmos. Sol.-Terr. Phy., in press, doi:10.1016/j.jastp2010.06.007, 2010.

Turner, J. M., Burlaga, L. F., Ness, N. F., and Lemaire, J. F.: Magnetic holes in the solar wind, J. Geophys. Res., 82, p. 1921, 1977.

Vasquez, B. J., Abramenko, V. I., Haggerty, D. K., and Smith, C. W.: Numerous small magnetic field discontinuities of Bartels rotation 2286 and the potential role of Alfvénic turbulence, J. Geophys. Res., 112, A11102, doi:10.1029/2007JA012504, 2007.

Vedenov, A. A. and Sagdeev, R. Z.: Some properties of a plasma with an anisotropic ion velocity distribution in a magnetic field, in: Plasma Phys. Prob. Controlled Thermo. Reacts., Pergamon, 3, p. 332, 1958.

Winterhalter, D., Neugebauer, M., Goldstein, B. E., Smith, E. J., Bame, S. J., and Balogh, A.: Ulysses field and plasma observations of magnetic holes in the solar wind and their relation to mirror-mode structures, J. Geophys. Res., 99, p. 23371, 1994.

Winterhalter, D., Neugebauer, M., Goldstein, B. E., Smith, E. J., Tsurutani, B. T., Bame, S. J., and Balogh, A.: Magnetic holes in the solar wind and their relation to mirror-mode structures, Space Sci. Rev., 72, p. 201, 1995.

Wu, C. S. and Davidson, R. C.: Electromagnetic instabilities produced by neutral-particle ionization in interplanetary space, J. Geophys. Res., 77, p. 5399, 1972.

Zwan, B. J. and Wolf, R. A.: Depletion of the solar wind plasma near a planetary boundary, J. Geophys. Res., 81, p. 1636, 1976. 